\title{
LO QUE CUENTAN LOS DOCUMENTOS: PARA UNA HISTORIA DEL ESPAÑOL DE CHILE EN EL PERÍODO COLONIAL*
}

\author{
WHAT RECORDS TELL: WRITING THE HISTORY OF \\ COLONIAL CHILEAN SPANISH
}

\section{MANUEL CONTRERAS SEITZ ${ }^{* * *}$}

\section{RESUMEN}

Si bien cada vez más el concepto de 'español atlántico' ha ido ganando fuerza como definición caracterizadora de un tipo de habla con isoglosas comunes, también es cierto que dentro del contexto hispanoamericano el enfoque no ha estado puesto en el Chile colonial precisamente, atención que sí han tenido otros países americanos. Una dificultad no menor ha sido la formación en lo que se consideraban 'ciencias auxiliares de la historia', pero que también forman parte del campo de la filología: paleografía y diplomática, principalmente. El empoderarse de una sociolingüística histórica, para la comprensión del rol característico de la comunidad hablante en el desarrollo del español del Chile colonial, resulta fundamental si queremos dar cuenta no sólo de las 'reglas' que rigen el cambio lingüístico en nuestra variante dialectal a través del tiempo, si no que, más importante aún, para recobrar la memoria. Trataré aquí de hacer una revisión de los trabajos que sirven de base para esta sociolingüística histórica del español de Chile, con algunas recomendaciones que pueden servir como punto de partida a la hora de recorrer la historia lingüística colonial, así como las dificultades que se presentan y de qué manera, desde mi experiencia, éstas pueden resolverse.

Palabras clave: Lingüística histórica, historia colonial, corpus de documentos coloniales.

* Este artículo se encuentra enmarcado en el Proyecto Fondecyt No 1130497, "La 'Conquista Espiritual del Reino de Chile’: una edición en capas del menologio jesuita de Diego de Rosales (transcripciones, análisis y ediciones)".

** Dr. en Filología Hispánica, Instituto de Lingüística y Literatura, Universidad Austral de Chile.Valdivia, Chile. Correo electrónico: manuelcontreras@uach.cl 


\section{ABSTRACT}

While increasingly the concept of 'Atlantic Spanish language' has been gaining strength as characterising definition of a type of speech with common isoglosses, it is also true that, within the Latin American context, colonial Chile has not enjoyed the same attention drawn to other countries. Training in paleography and diplomatics, once considered 'auxiliary sciences of history', but which are also part of the field of philology, has not been a minor problem. Knowledge of historical sociolinguistics for understanding the distinctive role of the speech community in the development of the Spanish language of colonial Chile, is essential if we are to account not only for the 'rules' governing linguistic change in our dialectal variant through time, but more importantly, to recover memory. In this article I try to review the works that are the basis for this historical sociolinguistics of the Spanish language in Chile, with some recommendations that can serve as a starting point when going over the colonial linguistic history, as well as the difficulties encountered and how, from my experience, they can be solved.

Keywords: Historical linguistics, colonial history, corpus of colonial records.

Recibido: 01.04.16. Aceptado: 16.06.16.

\section{INTRODUCCIÓN}

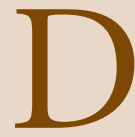

ENTRO DEL CAMPO de la lingüística histórica existen diversas vertientes, siendo quizá las más reconocidas aquellas que, en nuestro caso, hacen extensivas sus ramas hacia las lenguas clásicas (particularmente el latín, como fundamento de las lenguas romances) y hacia la historia del español peninsular. Si bien cada vez más el concepto de 'español atlántico' fue ganando fuerza como definición caracterizadora de un tipo de habla con isoglosas comunes, también es cierto que dentro del concierto hispanoamericano el acento no ha estado puesto en el Chile colonial precisamente, tanto por parte de nuestros investigadores como desde otros enclaves de nuestro Continente o de Europa, atención que sí han tenido otros países de la América hispana. Para los investigadores nacionales, creo, una dificultad no menor ha sido la formación en lo que se consideraban 'ciencias auxiliares de la historia', pero que también forman parte, como no, del campo de la filología: paleografía y diplomática, principalmente, pero también una sólida base -o un trabajo conjunto muy estrecho con especialistas- en la respectiva historia nacional.

El empoderarse de una sociolingüística histórica, para la comprensión del rol característico de la comunidad hablante en el desarrollo del español del Chile colonial, resulta fundamental si queremos dar cuenta no sólo de las 'reglas' que rigen la perspectiva del cambio lingüístico en nuestra va- 
riante dialectal a través del tiempo, ni tan sólo del contexto que rige dicho cambio, si no que, y creo más importante aún, para recobrar la memoria.

Trataré aquí de hacer una revisión de los trabajos que sirven de base para esta sociolingüística histórica del español de Chile, con algunas recomendaciones que, es probable, pueden servir como punto de partida (y también de llegada) a la hora de recorrer la historia lingüística colonial, así como las dificultades que se presentan y de qué manera, desde mi experiencia, éstas pueden salvarse.

La historia del español de Chile aún está por hacerse. Ha habido algunos avances, pero faltan dos pilares fundamentales: una mayor cantidad y diversidad de corpus documental transcrito, así como un mayor interés de los jóvenes lingüistas y filólogos por ahondar en este ámbito multidisciplinario.

\section{LINGÜÍSTICA HISTÓRICA: LINGÜÍSTICA DE CORPUS}

Como dijimos, cuando hablamos de lingüística histórica, pensamos tal vez en los romanistas desde Schuchardt hasta el mismo Eugenio Coseriu. Así, cabría preguntarse cuál puede ser nuestro aporte a esta disciplina, vista desde la perspectiva clásica. A lo que tendríamos que contestar, también probablemente, que poco. Y no por falta de entusiasmo, sino porque las herramientas con las cuales desempeñar esta labor filológica se encuentran cada vez más lejos. Porque no basta con un 'arsenal' teórico lingüístico sólido. Para hacer lingüística histórica, desde cualquier perspectiva, enfoque o cronología, debemos recurrir a los documentos, en toda la amplia gama estilística, para tratar de no perder óptica de la riqueza de la lengua sobre la cual hemos centrado el estudio.

Esto nos lleva, desde mi punto de vista, a varias cuestiones previas antes de plantearnos el análisis lingüístico del documento y, por ende, la caracterización de la variante dialectal en estudio: la determinación del corpus y de la manera en que éste debe ser transcrito. No es una discusión menor, pues el tipo de documento no sólo remite a posibilidades tipológicas, sino que también a la concentración de la variante sociolingüística que podemos ser capaces de desentrañar, por medio de las construcciones presentadas: es probable que en un documento personal hallemos, por ejemplo, una mayor frecuencia de uso de ciertas fórmulas de tratamiento que en un texto jurídico. Junto con ello, la elección del tipo de transcripción no dejará de ser fundamental para el tipo de estudio que nos hayamos planteado, puesto 
que no todo lo poco editado para el período colonial en Chile es de utilidad para un estudio lingüístico de nuestra variedad dialectal.

Plantearé -y trataré de mostrar- aquí algunas características de los trabajos que se efectúan con los documentos y los tipos de corpus que están sustentando el trabajo diacrónico en Hispanoamérica, en general, para situar el contexto chileno en particular

\subsection{La difícil realidad de la selección y transcripción del corpus}

Guillermo Guitarte, ya en 1968, planteaba la desventaja de la filología -particularmente la americana- respecto de la historia, en cuanto a la utilización de fuentes documentales para la investigación. Los textos literarios, en general, siempre han estado más a mano, sobre todo después de la imprenta, y tenemos que reconocer que algunas luces nos entregan sobre la realidad lingüística de la época; las gramáticas de diversa naturaleza, en algunas ocasiones, tienen testimonios valiosos y observaciones agudas sobre el habla de una determinada comunidad, sin embargo, nada reemplaza el trabajo con documentos de archivo, sean estos en papel o digitales, para enfrentar el trabajo filológico. Nuestro propio Archivo Nacional cuenta con una cantidad significativa de metros lineales de documentos coloniales, en distintos Fondos, que son de grandísima utilidad al investigador y que esperan por ser descubiertos.

Pero como dijimos anteriormente, los documentos que esperan allí no nos serán de utilidad en su 'estado propio', o en el mejor de los casos, como una imagen de alta resolución, sino que debemos hacerlos accesibles a quien va a analizarlos. Porque ésta es una cuestión previa, tal vez la de mayor relevancia, la más demandante y, también, la menos comprendida.

En primer término, creo, hay que situarse en la perspectiva para la cual se va a efectuar la transcripción: pudiera parecer fácil decir que, evidentemente, se trata de estudios lingüísticos, pero tal vez no tan fácilmente podríamos situarnos cómo debiera ser el tipo de texto con el cual vamos a trabajar a partir de esa primera definición. Por ejemplo, si tenemos la siguiente parte del folio 1r. (o todos los folios) del llamado Libro Becerro de las Actas del Cabildo de Santiago, ¿qué hacemos? 


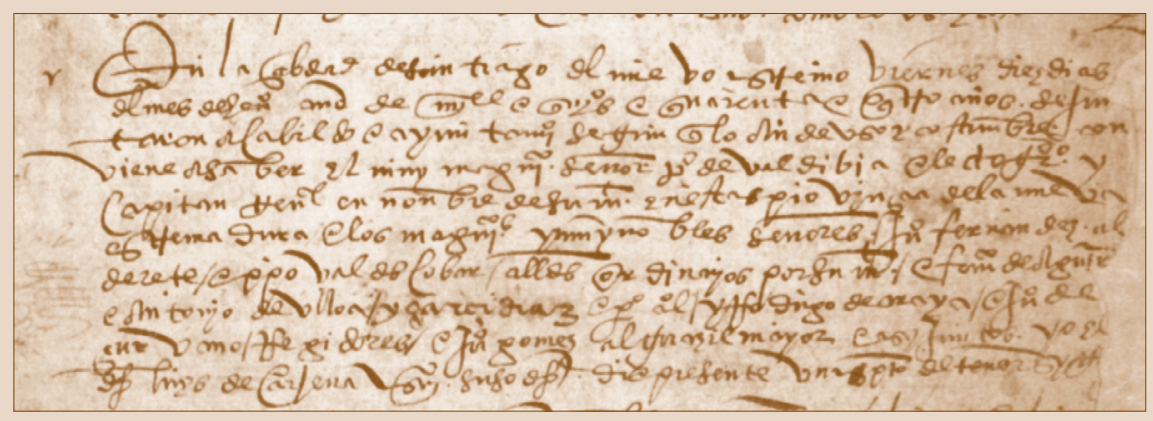

Una de las alternativas es seguir la pauta de Medina, quien transcribe este texto en el Tomo I de la Colección de Historiadores de Chile:

En la ciudad de Santiago del Nuevo Extremo, viérnes diez dias del mes de enero, año de mil e quinientos e cuarenta e cuatro años, se juntaron a cabildo e ayuntamiento, segun que lo han de uso y costumbre, conviene a saber: el mui magnífico señor Pedro de Valdivia, electo gobernador y capitan jeneral en nombre de S. M. en esta provincia de la Nueva Extremadura, e los magníficos e mui nobles señores Joan Fernandez Alderete, e Cristóbal de Escobar, alcaldes ordinarios por S. M., e Francisco de Aguirre, e Antonio de Ulloa, y Garci Diaz, e Pedro Alonso, y Rodrigo de Araya, e Joan de Curbano, rejidores, e Joan Gomez, alguacil mayor. E así juntos, yo el dicho Luis de Cartagena, escribano, susodicho dia presenté un escrito del tenor siguiente:

Otra alternativa, es realizar una transcripción paleográfica, como ésta (Contreras, 2013):

+En la çibdad de santiago del nuevo eotremo viernes diez dias del mes de henero año de mjll e qujnientos e quarenta e quatro añoб. se juntaron A Cabildo e ayuntamjento segun que lo An de uбo y coftunbre. conviene A saber El muy magnjfico señor. pedro de valdibja electo gobernador y Capitan general en nonbre de su magestad En ef ta ${ }^{1}$ provjnçia

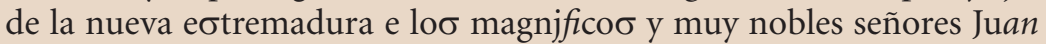
fernandez. alderete / e christoval ${ }^{2}$ descobar / allcaldes ordjnarjo $\sigma$ por su

\footnotetext{
${ }^{1}$ Tarjada una $-\mathrm{s}$

${ }^{2}$ Escrito "xpoval".
} 
magestad / e françisco de Agujr[re] e Antonjo de Vlloa / y garçi diaz e gonzalo alonso / y rrodrigo de araya / e Juan de çurvano / rregidores / e Juan gomes alguazil mayor e aбy juntoo. yo El dicho luyo de Carjena ${ }^{3}$ [sic] scribano suso dicho di e presente vn escripto del tenor oyguient[e]

En todo caso, podemos observar con cierta claridad varias dificultades en la lectura realizada por Medina que podrían incidir en un futuro análisis lingüístico: ciudad en vez de 'çibdad', extremo, Extremadura por 'eøtremo', 'eotremadura', Joan por 'Juan', Cristóbal por 'christoval', Joan de Curbano, en vez de 'Juan de çurvano'; Luis de Cartagena, escribano, susodicho dia presenté un escrito frente a 'luyo de Carjena ${ }^{4}$ [sic] scribano suso dicho di e presente vn escripto', lo cual nos remite, entre otras cosas, al cuestionamiento en relación con la puntuación de los textos coloniales.

Creo que hemos podido apreciar con cierta claridad cuál es el primer problema a la hora de hacer lingüística histórica, aun cuando sea para el período colonial chileno, en este caso ejemplificado en el siglo XVI.

Si bien podemos discutir la existencia de normas más o menos estrictas en cuanto al seguimiento de las grafías de este período, de ediciones críticas o anotadas, diplomáticas o semidiplomáticas, un primer tamiz es llegar a la comprensión del texto.

En la Biblioteca Antigua Chilena, a la cual ya haremos referencia, se encuentra establecida una serie de normas filológicas que se han tenido en cuenta en la edición documental; asimismo, se estableció en la colección de Documentos para la historia lingüistica de Hispanoamérica. Siglos XVI a XVIII que, en general, la transcripción iba a ser de carácter paleográfico estricto. En cuanto al corpus que he transcrito, en lo particular, he preferido un sistema mixto, semidiplomático, con despliegue de abreviaturas, para no caer en situaciones como la siguiente, que tomo como ejemplo de una carta de Isabel Mondragón (1569): " por amor de / dios yos lo rruego. q Las traygays aca porq A / ca las casare muy bien y ansi os lo rruega Luis to //".

¿Quién es Luis to? ¿Testigo, testamento, tesorero, tomín? Demasiadas interpretaciones para un lector común y aun para un lingüista que desee implicarse en el estudio histórico de nuestra variante dialectal. De allí que exista la necesidad profunda del trabajo interdisciplinario efectivo y sin condiciones, con humildad pero a la vez constante.

\footnotetext{
3 "Cartajena”.

4 "Cartajena”.
} 
En todo caso, para que podamos salir de la duda, en el folio siguiente, en las líneas 8 y 9, dice: "Luis ternero y maria mondra / gona vra prima os besan las $\mathrm{ms}^{\mathrm{o}}$ ".

\subsection{Los corpus hispanoamericanos y chilenos}

En cuanto al otro factor, la existencia y producción de corpus para el análisis, fundamental a la hora de poder decir algo respecto de la conformación del español americano, y de Chile en particular, no es menos cierto que las realidades son completamente diferentes.

De suyo, existen zonas dialectales que están sumamente representadas, ya que la tradición escrituraria y también filológica han sido un punto valioso en la configuración cultural de las mismas: México, Perú, Argentina, Colombia, Costa Rica, Uruguay, etc. De particular interés son las iniciativas de la Real Academia Española, a través del CORDE (Corpus Diacrónico del Español) y de la Academia Mexicana de la Lengua, por medio del trabajo del CORDIAM (Corpus Diacrónico y Diatópico del Español de América).

El primero, disponible a través de la página de la RAE (http://corpus. rae.es/cordenet.html), es una recopilación de diversa naturaleza, desde los orígenes hasta 1974, fecha en que comienza el CREA (Corpus de Referencia del Español). En lo que se refiere a Chile, en todo caso, hay una muy pobre representación, pues sólo están presentes los textos que forman parte de los DHLH, esto es, 47 documentos entre los siglos XVI y XVIII.

En el ámbito hispanoamericano ha habido numerosas iniciativas, tanto de colecciones de textos como de corpus. Recientemente, Iberoamericana/ Vervuert ha dedicado una serie de Textos y documentos Españoles y Americanos, que incluyen, hasta el momento, 8 volúmenes, de los cuales los 7 primeros están conformados por testimonios de archivo 5 :

-Rivarola, José Luis (2000). Español Andino. Textos de bilingües de los siglos XVI y XVII. (30 textos, entre 1587 y 1679).

-Stoll, Eva (2002). La Memoria de Juan Ruiz de Arce. Conquista del Perú, saberes de caballería y defensa del mayorazgo. (Edición diplomática, texto c.1543, 17 folios por ambas caras).

${ }^{5}$ La octava obra es: Diez del Corral Areta, Elena (2015). Los conectores consecutivos en documentos coloniales de la Audiencia de Quito (1563-1822). Se analiza un total de 381 documentos, pero que no están anexados a la obra. 
-Schmidt-Riese, Roland (2003). Relatando México. Cinco textos del período fundacional de la colonia en Tierra Firme. (Estudio y edición de manuscritos entre 1540 y 1560).

-Navarro Gala, Rosario (2007). La "Relación de antigüedades deste Reyno del Pirú". Gramática y discurso ideológico indígena. (Texto de Joan de Santa Cruz Pachacuti Yamqui, siglo XVII; 43 folios).

-Albertin, Chiara (ed.) (2008). De las costumbres antiguas de los naturales del Pirú. (Edición paleográfica, texto fines siglo XVI o ppios. XVII). -Fernández Alcaide, Marta (2009). Cartas de particulares en Indias del siglo XVI. Edición y estudio discursivo. (640 documentos, anexados en un $\mathrm{CD}$ ).

-Stoll, Eva y Ma de las Nieves Vázquez Núñez (eds.) (2011). Alonso Borregán. La Conquista del Perú. (Edición diplomática, texto del siglo XVI).

Una interesante iniciativa, con textos principalmente de naturaleza literaria, se está llevando a cabo a través del proyecto de colección El Paraíso en el Nuevo Mundo, liderado por el Dr. Ramón Manuel Pérez Martínez, de la Universidad San Luis Potosí (México). La iniciativa constará de textos coloniales, en ediciones críticas o anotadas, dirigidas a un público semiespecializado y pluridisciplinar.

En cuanto a la recopilación de corpus hispanoamericano, a partir del trabajo de diversas comisiones de ALFAL, y por cierto de distintos equipos en los países americanos y en España, surge la iniciativa del CORDIAM (Corpus Diacrónico y Diatópico del Español de América), que se "alimenta", en principio, de una serie de documentos ya publicados en diversas fuentes:

-Academia Nacional de Letras del Uruguay, Documentos para un corpus diacrónico del español del Uruguay, Juan Justino da Rosa (coord.), con la colaboración de Eloísa Cajaraville, Juan Carlos Urse, Soraya Ochoviet, Rosa Chans, Stella Almirón, Adriana Cabakián. Transcripciones inéditas. -Edward Baranowski, Documents from the 1602-3 Sebastián Vizcaino Expedition up the California Coast, Cíbola Project, Research Center for Romance Studies, California: University of California Berkeley.

-Virginia Bertolotti, A mí de vos no me trata ni usted ni nadie. Sistemas e historia de las formas de tratamiento en la lengua española en América, México: Universidad Nacional Autónoma de México-Universidad de la República, 2015.

-Virginia Bertolotti, Magdalena Coll y Ana Clara Polakof, Documentos para la historia del español en el Uruguay. Vol. 1. Cartas personales y do- 
cumentos oficiales y privados del siglo xviii, Montevideo: Facultad de $\mathrm{Hu}-$ manidades y Ciencias de la Educación, 2010.

-Virginia Bertolotti, Magdalena Coll y Ana Clara Polakof, Documentos para la historia del español en el Uruguay. Vol. 2. Cartas personales y documentos oficiales y privados del siglo xix, Montevideo: Facultad de Humanidades y Ciencias de la Educación, 2012.

-Micaela Carrera de la Red, Corpus de Colombia. Transcripciones inéditas. -Belem Clark de Lara y Concepción Company Company, Lengua, cultura y literatura en el siglo xvii en México. Materiales para su estudio. Transcripciones inéditas.

-Concepción Company Company, Documentos lingüísticos de la Nueva España. Altiplano central, México: Universidad Nacional Autónoma de México, 1994.

-Ana María Díaz Collazos y Yamileth Ortiz Vanegas, Documentos de la Audiencia de Santa Fe de Bogotá, siglo xvi.Transcripciones inéditas.

-José María Enguita Utrilla, Relación de las fábulas y ritos de los Ingas, de Cristóbal de Molina. Transcripción inédita.

-María Cristina Egido, Documentos del oriente de Bolivia (siglos xvii-xviii). Transcripciones inéditas y versión revisada de "iii: Bolivia-b", en E. Rojas (ed.), Documentos para la historia lingüistica de Hispanoamérica. Siglos xvi a xviii, anejo lx del Boletín de la Real Academia Española, Madrid: Real Academia Española, 2008, pp. 457-483.

-Marta Fernández Alcaide, Cartas de particulares en Indias del siglo xvi. Edición y estudio discursivo, libro y CD Rom, Madrid / Frankfurt: Iberoamericana / Vervuert, 2009.

-Martha Guzmán, Documentos del siglo xv al xvii de las Antillas. Transcripciones inéditas.

-Ofelia Huamanchumo de la Cuba, "Apéndice D. Visita: 1549 Guanca", en Encomiendas y cristianización. Análisis pragmático de documentos jurídicos y administrativos del Perú (Siglo xvi), München: Mikroform KarlHeinz Limbeck, 2011.

-María del Carmen Martínez Martínez, Desde la otra orilla. Cartas de Indias en el Archivo de la Real Chancillería de Valladolid (siglos xvi-xviii), edición, estudio, notas e índices, León: Universidad de León, 2007.

-Mariela Masih, Cartas coloniales. Córdoba (Argentina). Siglos xvi-xvii, Córdoba: Babel, 2009.

-Chantal Melis, Agustín Rivero Franyutti, con la colaboración de Beatriz Arias Álvarez, Documentos lingüísticos de la Nueva España. Golfo de México, México: Universidad Nacional Autónoma de México, 2008. 
-José G. Mendoza (coordinador), 100 documentos para la historia lingüística de Bolivia. Siglos xvi-xviii (Alto Perú), con la colaboración de Ofelia Moya, E. Lía Pinto Yarvi, Judith Céspedes, María Luz Gómez Sumi, Lillet Jovana Huanca Ortuño, Luz Jimena López Roberts, Javier Paredes Mallea, Marcela E. Pinto Diamantino, Rosario Salas, España Rosario Villegas Pinto e Irma Paula Yampasi, La Paz: Universidad Mayor de San Andrés, 2000.

-Enrique Obediente Sosa, Textos para la historia del español. Mérida, Venezuela (siglos xviii-xix). Transcripciones inéditas.

-Miguel Ángel Quesada Pacheco, Fuentes documentales para el estudio del español colonial de Costa Rica, San José: Alma Mater, 1987.

-Claudia Parodi (coordinadora), Crónica de Chimalhuacán, transcripción de Claudia Parodi, Helen Ellis, Aarón A. Olivas, Bryan Green, Ángela Helmer, Alicia Houtrow, Miriam Villanueva, Lizi Moromisato, Belén Villareal y Jimena N. Rodríguez, Centro de Estudios Coloniales Iberoamericanos de la University of California Los Angeles. Transcripción inédita.

-Vicente Juan Pérez Sáez y María Fanny Osán de Pérez Sáez, El español de la Argentina. Documentos para su historia (Noroeste), con la colaboración de Silvia Torino, 2 volúmenes, Salta: Impresora Gofica, 1997.

-Ana María Postigo de De Bedia y Lucinda Díaz de Martínez, Documentos del Jujuy colonial. Aportes para el estudio histórico del español americano (siglos xvi a xix), San Salvador de Jujuy: EdiUnJu.

-José Luis Ramírez Luengo, Textos para la historia del español. Honduras (siglos xvii-xix). Transcripciones inéditas.

-José Luis Ramírez Luengo, "Un corpus para la historia del español en Nicaragua: edición de documentos oficiales del siglo xviii (1704-1756)", Moenia, 17, 2011, pp. 333-366.

-José Luis Ramírez Luengo, Textos para la historia del español. El Salvador (siglos xvii-xix). Transcripciones inéditas.

-Pedro Ángel Ramírez Quintana, Documentos lingüísticos de la Nueva España. Provincia de Campeche, México / Campeche: Universidad Nacional Autónoma de México / Universidad Autónoma de Campeche, 2015. -Research Center for Romance Studies (University of California Berkeley), Cibola Project, Jerry R. Craddock y Barbara De Marco (coordinadores), https://escholarship.org/uc/rcrs_ias_ucb_cibola.

-Paloma Paula Reyna Vázquez, El siglo xviii en el Altiplano central de México. Materiales para su estudio. Edición crítica, estudio filológico, introducción y notas, tesis de licenciatura inédita, México: Universidad Nacional Autónoma de México, 2006. 
-José Luis Rivarola, Documentos lingüísticos del Perú. Siglos xvi y xvii. Edición y comentario, Anejo 103 de la Revista de Filología Española, Madrid, Consejo Superior de Investigaciones Científicas, 2009.

-Agustín Rivero Franyutti, Aproximación al español mexicano en el siglo xvi: edición crítica y estudio filológico de un conjunto de cartas (15371557), tesis de doctorado inédita, México: Universidad Nacional Autónoma de México, 2000.

-Elena Rojas Mayer (compiladora y editora), Documentos para la historia lingüistica de Hispanoamérica. Siglos xvi-xviii, tomo ii, Anejo lviii del Boletín de la Real Academia Española, Madrid: Real Academia Española, 2000.

-Elena Rojas Mayer (compiladora y editora), Documentos para la historia lingüística de Hispanoamérica. Siglos xvi-xviii, tomo iii, Anejo lxi del Boletín de la Real Academia Española, Madrid: Real Academia Española, 2008.

-Israel Sanz-Sánchez, Documentos de los Spanish Archives of New Mexico. Transcripciones inéditas.

-Israel Sanz-Sánchez, The Diachrony of New Mexican Spanish, 16831926: Philology, Corpus Linguistics and Dialect Change, tesis de doctorado inédita, Berkeley: University of California Berkeley, 2009.

-Luciana de Stefano y María Josefina Tejera, Documentos para la historia del español de Venezuela, siglos xvi-xviii, Caracas: Universidad Central de Venezuela, 2006. Publicación en CD.

-Ana Tsutsumi, Documentos lingüísticos de la Nueva España: Oaxaca, Chiapas y Guatemala, con la colaboración de Bangladesh Ríos, Mariana Orozco y Concepción Company. Transcripciones inéditas.

-Nerea Zabalegui (coordinadora), Corpus de documentos escritos en Venezuela (siglos xviii y xix), Laura Silva, Erika Contreras, María Elena Castejón, Víctor Osorio y Nerea Zabalegui (investigadores y editores). Transcripciones inéditas.

Como podemos apreciar, la representatividad chilena aquí era nula.

\section{LA FILOLOGÍA EN CHILE Y LOS CORPUS DIACRÓNICOS}

La filología chilena ha pasado por diversos derroteros con preocupaciones, ya desde los inicios de la República, relacionadas con el desarrollo de los usos ortográficos (particularmente en la discusión sobre la norma que de- 
bería estandarizarlos), los estudios lexicológicos y lexicográficos (principalmente enfocados en la corrección idiomática y en los usos diferenciales), las obras dedicadas a las lenguas indígenas (con especial dedicación al 'araucano'), entre otros. Con todo, Rojas (1940, p. 171) -quien establece una guía crítica de los estudios filológicos en Chile, desde el período colonial hasta fines de la década de 1930, con lo cual permite una panorámica completa del conocimiento generado hasta ese entonces, refiriendo no sólo las obras o autores de mayor connotación, sino todos aquellos que publicaron sus trabajos vinculados con la temática en estudio, obras que clasifica a partir de la lectura exhaustiva de las mismas- al referirse a los estudios sobre gramática y métrica históricas, ya señalaba en esta época que:

Esta rama de los estudios filológicos, que bien pudiéramos considerar como fundamental, ha sido poco cultivada en nuestro país, no sabemos si porque ella exige una dedicación permanente y una profunda versación en asuntos lingüísticos, o por requerir un cabal conocimiento del latín y aun del griego, idiomas cuyo estudio se ha abandonado en Chile desde hace años.

Bien puede extrapolarse esta situación a las ediciones de textos, particularmente las del período colonial chileno ${ }^{6}$, las que parecen haber experimentado parte de la animosidad con la cual se ha mirado, y aún se sigue observando, el periodo histórico vinculado con la Corona Española en Hispanoamérica. Ha sido, más bien, desde el interés histórico que se rescataron algunos textos, en principio, como la Colección de Documentos Inéditos para la Historia de Chile, de José Toribio Medina; la Crónica y Relación Copiosa y Verdadera de los Reinos de Chile, en transcripción de Irving Leonard (1966); las Fuentes para la Historia del Trabajo en el Reino de Chile, de Álvaro Jara y Sonia Pinto (1982-1983), las Cartas de Mujeres en Chile. 1630-1885, de Sergio Vergara (1987), los Protocolos de los Escribanos de Santiago, de Álvaro Jara y Rolando Mellafe (1996), los Testamentos de Indios en Chile Colonial, de Julio Retamal (2000), o también el valioso esfuerzo que realizó la Editorial Universitaria, en su colección de Escritores Coloniales de Chile, en la cual se difundieron fragmentos de obras literarias de dicho periodo. Con todo, el esfuerzo de versiones filológicas recién comienza con el trabajo de Mario Ferreccio, quien inaugura la llamada Biblioteca Antigua de Autores Chilenos

\footnotetext{
${ }^{6}$ Góngora Marmolejo, Alonso de (1575). Historia de todas las cosas que han acaecido en el reino de Chile y de los que lo han gobernado. Estudio, edición y notas de Miguel Donoso Rodríguez, Iberoamericana/Vervuert, Madrid-Frankfurt, 2010.
} 
[luego Biblioteca Antigua Chilena, BACh] con una edición crítica del Purén Indómito, donde se refieren exhaustivamente los criterios abordados para el estudio y la edición misma del manuscrito. Hasta la fecha, la BACh la constituyen:

-Purén Indómito. Diego Arias de Saavedra (prólogo y edición crítica de Mario Ferreccio; estudio preliminar de Mario Rodríguez).

-Relación autobiográfica. Úrsula Suárez (prólogo y edición crítica de Mario Ferreccio; estudio preliminar de Armando de Ramón).

-El Ignacio de Cantabria. Pedro de Oña (edición crítica de Mario Ferreccio).

-La Guerra de Chile. Anónimo (edición crítica de Mario Ferreccio y Raïssa Kordić).

-Cautiverio feliz. Francisco Núñez de Pineda y Bascuñán (edición crítica de Mario Ferreccio y Raïssa Kordić).

-Testamentos coloniales chilenos. AA.VV. (edición crítica a cargo de Raïssa Kordić. Son 54 testamentos, 3 poderes para testar, 2 codicilos, una carta de donacion entre vivos, una carta de perdón y una memoria, esto es, 62 documentos entre 1593 y 1697).

-Epistolario de Sor Dolores Peña y Lillo (Chile, 1763-1769) (edición crítica a cargo de Raïssa Kordić).

No hay que olvidar, en todo caso, que en 1991, con motivo de la celebración de los 500 años del Encuentro de Dos Mundos, la barcelonesa editorial Lumen publicó el libro: Cartas de Don Pedro de Valdivia que tratan del descubrimiento y conquista de la Nueva Extremadura, en edición facsimilar y con la transcripción del destacado filólogo chileno. Asimismo, la editorial Pehuén publica nuevamente, en el 2003, la edición crítica de la Histórica relación del Reino de Chile, de Alonso de Ovalle, versión que Mario Ferreccio había realizado en 1969 para el Instituto de Literatura Chilena. El año 2001, Alamiro Ávila y Lucía Invernizzi nos entregan una versión de la Historia Alonso de Góngora Marmolejo; para el 2010, Miguel Donoso publicó en Iberoamericana una nueva y excelente edición crítica de la misma obra ${ }^{7}$, ampliando los horizontes de la misma. El editor señala, respecto de la anotación filológica -que nos interesa en la discusión- que:

\footnotetext{
${ }^{7}$ Otte (1996) sólo contempla 4 cartas chilenas del XVI: 3 de Isabel Mondragón y una de María Mondragón.
} 
Se ha insistido muchas veces en que es imposible disociar la ecdótica de la hermenéutica. Para fijar bien un texto hay que entenderlo y este no se puede entender si está mal fijado. Pero para entender el texto es vital además la tarea de interpretación del mismo, la cual se materializa en el aparato de notas explicativas a pie de página.

Como parte de una inquietud personal, esta vez, inicié un camino para conformar lo que, siguiendo los parámetros establecidos por ALFAL, se transformaría en el Corpus Diacrónico del Español de Chile (CorDECh), compuesto por los 47 textos corregidos y en versión semidiplomática de "Santiago de Chile", que conformaron los DHLH, y una versión adicionada de 242 documentos, entre los siglos XVI y XVIII. Esta muestra textual se encuentra disponible en los 3 volúmenes que conforman la colección $\mathrm{Me-}$ moria documental en textos chilenos del período colonial.

El volumen correspondiente al siglo XVI consta de 89 documentos, encabezados por la Nueva obra y breve, en prosa y metro, sobre la muerte del Ilustre Señor Adelantado Don Diego de Almagro, de los cuales 19 pertenecen a los DHLH. En cuanto a los del siglo XVII, la muestra alcanza los 117 documentos (15 de los DHLH); se compone esta serie por 39 transcripciones correspondientes al primer volumen del Archivo Contaduría Mayor, Primera Serie. Finalmente, en el siglo XVIII, tenemos 83 manuscritos -13 de los cuales ya estaban en los DHLH-, se agrega a esta muestra la transcripción íntegra de las Informaciones de Nobleza que para Caballero de la Orden Militar de Santiago se hicieron de Don Diego Calvo de Encalada y Orozco, natural de la ciudad de Sevilla.

En cuanto al corpus inédito, el CorDECh está compuesto por 718 fojas, de 181 documentos de 7 volúmenes del Fondo Real Audiencia, que van entre los años de 1628 a 1788. Este corpus se encuentra en proceso de revisión para ser publicado. Asimismo, se trabaja en la edición de la Conquista espiritual del Reino de Chile, de Diego de Rosales, manuscrito del siglo XVII de 429 fojas, a doble columna. Esto es, al término de la edición del fondo mencionado, se contaría con un total de casi 300 documentos.

Cabe señalar que ya este material está siendo incorporado en el CORDIAM.

\section{COROLARIO}

Si comparamos la proporción documental con otra realidades americanas, nuestro Archivo Nacional parece algo modesto. Sin embargo, la escasa pro- 
ducción de ediciones diplomáticas o semidiplomáticas, anotadas o críticas, hace que sea necesario dimensionar la magnitud de la tarea pendiente, particularmente en lo referido al primer siglo de nuestra historia colonial (1540-1650).

Por ejemplo, un fondo particularmente interesante, por la diversidad de los documentos que contiene, es el de Escribanos de Santiago, que va de 1579 a 1800 , y que comprende 973 volúmenes. Si calculamos una media de 400 fojas por volumen, sólo este cuerpo textual tendría alrededor de 380.000 fojas. En cuanto a la transcripción, también en promedio, si se estima alrededor de 20 minutos por foja, esto es, 3 fojas por hora, le llevaría a una persona 127.000 horas (5.291 días, o mejor, 1411/2 años) transcribir toda este ingente corpus. Claro que aquí no se considera la preparación de una edición crítica o anotada, sino sólo la transcripción y digitación del respectivo texto. Aunque no es el caso aquí, tal vez el trabajo interdisciplinario no sólo con historiadores, sino también con ingenieros informáticos, puede llevarnos al desarrollo de una 'paleografía digital', como se hace en otras lenguas, para facilitar en parte un primer acercamiento al documento.

Mi propuesta, en este sentido, es simple de formular, pero requiere del interés y de la constancia de muchos actores: compartir la información y dividir el trabajo, esto es, centralizar aquello que estamos haciendo para no repetir esfuerzos, utilizar criterios comunes para la edición documental y trabajar en conjunto para lograr constituir un corpus lo suficientemente amplio para dar cuenta del desarrollo histórico de nuestra variedad dialectal. El enorme potencial de las redes sociales y de las comunidades virtuales es hoy nuestro reto. Se requiere formar "escuela", comunidades de aprendizaje y de trabajo.

\section{REFERENCIAS}

Contreras, M.E. (2013). Memoria documental en textos chilenos del período colonial, Vol. I. Edición semidiplomática, siglo XVI. Saarbrücken, Alemania: Editorial Académica Española.

Contreras, M.E. y Salvo, M. (2013). Memoria documental en textos chilenos del período colonial, Vol. II. Edición semidiplomática, siglo XVII. Saarbrücken, Alemania: Editorial Académica Española.

Contreras, M.E., Jara, R. y Molina, R. (2013). Memoria documental en textos chilenos del período colonial, Vol. III. Edición semidiplomática, siglo XVIII. Saarbrücken, Alemania: Editorial Académica Española.

Coseriu, E. (1954). El llamado 'latín vulgar' y las primeras diferenciaciones ro- 
mances: breve introducción a la lingüística románica. Montevideo, Uruguay: Universidad de la República.

Guitarte, G. (1968). "Para una historia del español de América basado en documentos: El seseo en el Nuevo Reino de Granada (1550-1650)". Actas de la $5^{a}$ Asamblea Interuniversitaria de Filología y Literatura Hispánicas, 158-165, Bahía Blanca, Argentina: Universidad Nacional del Sur.

Jara, A. y Mellafe, R. (1996). Protocolos de los escribanos de Santiago. Primeros fragmentos, 1559 y 1564-1566. Santiago, Chile: Centro de Investigaciones Diego Barros Arana.

Jara, A. y Pinto, S. (1982-1983). Fuentes para la historia del trabajo en el Reino de Chile, Legislación, 1546-1810, tomos I y II. Santiago, Chile: Andrés Bello.

Medina, J. T. (1888-1902). Colección de documentos inéditos para la historia de Chile, desde el viaje de Magallanes hasta la Batalla de Maipú (1518-1818), 30 vols. Santiago, Chile: Ercilla - Elzeviriana.

Otte, E. (1996). Cartas privadas de emigrantes a Indias. 1540-1616. México D.F., México: Fondo de Cultura Económica.

Real Academia Española (1993). Documentos para la historia lingüistica de Hispanoamérica. Siglos XVI a XVIII, Anejo LIII BRAE. Madrid, España: Espasa-Calpe. . (2000). Documentos para la historia lingüística de Hispanoamérica. Siglos XVI a XVIII, II, Anejo LVIII BRAE. Madrid, España: Espasa-Calpe. . (2008a). Documentos para la historia lingüística de Hispanoamérica. Siglos XVI a XVIII, III, Anejo LX BRAE. Madrid, España: Espasa-Calpe. . (2008b). Documentos para la historia lingüística de Hispanoamérica. Siglos XVI a XVIII, IV, Anejo LXI BRAE. Madrid, España: Espasa-Calpe.

Retamal, J. (2000). Testamentos de indios en Chile colonial. Santiago, Chile: RIL. Rojas, G. (1940). Filología chilena. Guía bibliográfica y crítica. Santiago, Chile: Imp. y Lit. Universo, S.A.

Schuchardt, H. (1866). Der Vokalismus des Vulgärlateins. Leipzig, Alemania: Druck und Verlag von B. G. Teubner.

Vergara, S. (1987). Cartas de mujeres en Chile, 1630-1885. Santiago, Chile: Andrés Bello.

Vivar, Jerónimo de (1966 [1558]). Crónica y relación copiosa y verdadera de los Reynos de Chile, transcripción paleográfica de Irving Leonard. Santiago, Chile: Fondo Histórico y Bibliográfico José Toribio Medina. 